PROCEEDINGS OF THE

AMERICAN MATHEMATICAL SOCIETY

Volume 129, Number 3, Pages 647-655

S 0002-9939(00)05597-0

Article electronically published on September 19, 2000

\title{
HIGHER RELATIVE PRIMITIVE IDEALS
}

\author{
GUANGFENG JIANG AND ARON SIMIS
}

(Communicated by Wolmer V. Vasconcelos)

\begin{abstract}
The main object of this note is to introduce a higher order ana$\log$ of the so-called primitive ideal of $Y$ relative to $X$ introduced by JiangPellikaan-Siersma, where $X \supset Y$ are germs of analytic subspaces of $\left(\mathbb{C}^{n}, 0\right)$. Our treatment of the problem is ideal-theoretic throughout, using the notion of iterated higher differential operators. Some examples from singularity theory are worked out. We establish the connection between higher primitive ideals and (relative) symbolic powers of an ideal and give an effective algorithm to compute both.
\end{abstract}

\section{INTRODUCTION}

Let $X \supset Y$ be germs of analytic subspaces of $\left(\mathbb{C}^{n}, 0\right)$, defined by ideals $\mathfrak{h} \subset \mathfrak{g} \subset$ $\mathcal{O}=\mathcal{O}_{\mathbb{C}^{n}, 0}$, respectively. Typically, one assumes that $X$ and $Y$ are reduced and pure dimensional. The main object of this note is to introduce a higher order analog of the so-called primitive ideal of $Y$ relative to $X$ introduced by Jiang-PellikaanSiersma (cf. 6], [7, 2]).

On the geometric level, the primitive ideal of $Y$ in $X$ consists of all functions whose zero level hypersurfaces pass through $Y$ and are tangent to $X_{\text {reg }}=X \backslash X_{\text {sing }}$ along $Y \cap X_{\text {reg. }}$. Our present notion of higher (iterated) primitive ideal of $Y$ collects those functions whose zero level hypersurfaces pass through $Y$ and have higher order of contact with $X$ along $Y \cap X_{\text {reg }}$. There are actually two notions of higher primitive ideals in the relative case due to the fact that higher (algebraic) differential operators on a singular space are not just the iterated composites of differential operators of order one. Since this intrinsic difficulty leads to a few delicate points in the theory, we made a choice to work with the iterated version.

In the absolute case, i.e., when $X=\mathbb{C}^{n}$, the higher primitive ideals coincide with the higher symbolic powers (phased out by one) of the ideal $\mathfrak{g}$ - this is a consequence of the well-known Nagata-Zariski theorem on holomorphic functions. The second symbolic power has shown quite useful in some of the earlier work in singularity theory. Of course, symbolic powers have been thoroughly studied in commutative algebra ever since they were formally introduced by W. Krull in

Received by the editors March 18, 1999 and, in revised form, May 12, 1999.

2000 Mathematics Subject Classification. Primary 13N15, 14B05; Secondary 13N10, 13P99, $16 \mathrm{~S} 32,32 \mathrm{~S} 05$

Key words and phrases. Primitive ideal, symbolic power, differential operator, Jacobian matrix, surface singularity.

The first author was supported by JSPS: P98028, and the second author was partially supported by CNPq, Brazil. 
the early thirties. It therefore seems quite natural to ask if the present notion of higher primitive ideals in the relative case $\mathfrak{h} \subset \mathfrak{g}$ likewise bears a close relation to the higher symbolic powers of the ideal $\mathfrak{g} / \mathfrak{h}$ in the residue class ring $\mathcal{O} / \mathfrak{h}$. This approach was taken in [2] in the case of the primitive ideal of order one. This paper deals systematically with this question for higher order. We also show that the higher (iterated) primitive ideals are effectively computable in terms of syzygies of certain matrices. As a bonus, we answer under certain conditions a question posed by Vasconcelos as to how to iteratively compute (relative) symbolic powers in an effective way (cf. [10] Section 3.5] for a survey of some methods).

The authors are indebted to the referee for pointing out several mistakes in an earlier version of this work.

\section{Preliminaries on differential operators}

Let $\mathcal{O}$ be a commutative ring and let $k \subset \mathcal{O}$ be a subring. The set $\operatorname{End}_{k}(\mathcal{O})$ of $k$-module endomorphisms of $\mathcal{O}$ is an $\mathcal{O}$-module in the usual way, whereby scalar multiplication $a \rho\left(a \in \mathcal{O}, \rho \in \operatorname{End}_{k}(\mathcal{O})\right)$ is to be taken as the composite $\mu_{a} \circ \rho$, with $\mu_{a}$ denoting the endomorphism multiplication by $a$.

Given an integer $r \geq 0$, let $\operatorname{Diff}^{(r)}(\mathcal{O})=\operatorname{Diff}_{k}^{(r)}(\mathcal{O}) \subset \operatorname{End}_{k}(\mathcal{O})$ denote the $\mathcal{O}$ submodule consisting of the differential operators of order $\leq r$ of the $k$-algebra $\mathcal{O}$. The traditional reference for differential operators of algebras is [1] (cf. also [5]).

Our main interest will stem from the following notion.

Definition 1. Let $\mathfrak{h} \subset \mathcal{O}$ be an ideal. The rth differential idealizer of $\mathfrak{h}$ is

$$
\mathbb{I}_{\mathfrak{h}}^{(r)}:=\left\{\delta \in \operatorname{Diff}^{(r)}(\mathcal{O}) \mid \delta(\mathfrak{h}) \subset \mathfrak{h}\right\}
$$

If no confusion arises, we will refer to the $r$ th differential idealizer simply as the idealizer (of order $r$ ). From the structure of the $\mathcal{O}$-module of $\operatorname{End}_{k}(\mathcal{O})$ as above, it easily follows that the idealizer of order $r$ of $\mathfrak{h}$ is an $\mathcal{O}$-submodule of $\operatorname{Diff}{ }^{(r)}(\mathcal{O})$. For $r=0$, $\operatorname{Diff}^{(r)}(\mathcal{O})=\mathcal{O}$. Thus, $\mathbb{I}_{\mathfrak{h}}^{(0)}=\mathcal{O}$. Since $\operatorname{Diff}^{(r)}(\mathcal{O})=\mathcal{O} \oplus \operatorname{Der}^{(r)}(\mathcal{O})$, where $\operatorname{Der}^{(r)}(\mathcal{O})$ is the $\mathcal{O}$-module of $k$-derivations of order $r$ of $\mathcal{O}$, then $\mathbb{I}_{\mathfrak{h}}^{(r)}=\mathcal{O} \oplus \widehat{\mathbb{I}_{\mathfrak{h}}^{(r)}}$, where $\widehat{\mathbb{I}_{\mathfrak{h}}^{(r)}}=\left\{\delta \in \operatorname{Der}^{(r)}(\mathcal{O}) \mid \delta(\mathfrak{h}) \subset \mathfrak{h}\right\}$. The latter module is the essential part of the differential idealizer. Throughout we set $\mathbb{I}_{\mathfrak{h}}=\mathbb{I}_{\mathfrak{h}}^{(1)}$.

Remark 2. In the case where $k=\mathbb{C}$ and $\mathcal{O}$ is a polynomial ring $\mathbb{C}\left[z_{1}, \ldots, z_{n}\right]$ (resp. a power series ring $\mathbb{C}\left\{z_{1}, \ldots, z_{n}\right\}$ ), interpreting $\operatorname{Der}(\mathcal{O})=\operatorname{Der}^{(1)}(\mathcal{O})$ as (algebraic) vector fields on the affine space $\mathbb{C}^{n}$ (resp. germs of analytic vector fields at $0 \in \mathbb{C}^{n}$ ), then the essential part of the differential idealizer of order 1 of $\mathfrak{h}$ is to be interpreted as the module of vector fields tangent to the subvariety $V \subset \mathbb{C}^{n}$ (resp. to the germ $(V, 0)$ of the analytic subspace at 0$)$ defined by the ideal $\mathfrak{h}$. In the case where $V$ is a hypersurface (a divisor), these vector fields were dubbed logarithmic vector fields along $V$ (cf. [8]). For higher values of $r$, (the essential part of) the differential idealizer yields a notion of vector fields of higher order of contact along $V$.

For a given $r \geq 0$, we consider yet another $\mathcal{O}$-module, namely, the $\mathcal{O}$-submodule $\left(\mathbb{I}_{\mathfrak{h}}\right)^{r} \subset \mathbb{I}_{\mathfrak{h}}^{(r)}$ generated by the elements of $\mathbb{I}_{\mathfrak{h}}$. In other words, the elements of this submodule are the $\mathcal{O}$-linear combinations of composites of $t$ elements of $\mathbb{I}_{\mathfrak{h}}$ for values $t \leq r$. Clearly, for $r \leq 1$ the two modules coincide. A similar remark takes 
place regarding the essential part of the latter module as defined in terms of partial composites of ordinary derivations.

For a fixed element $f \in \mathcal{O}$, the sets $\left(\mathbb{I}_{\mathfrak{h}}\right)^{r}(f):=\left\{\delta(f) \mid \delta \in\left(\mathbb{I}_{\mathfrak{h}}\right)^{r}\right\} \subset \mathbb{I}_{\mathfrak{h}}^{(r)}(f):=$ $\left\{\delta(f) \mid \delta \in \mathbb{I}_{\mathfrak{h}}^{(r)}\right\}$ are ideals of $\mathcal{O}$. The fact that these are ideals has solely to do with the nature of $\mathbb{I}_{\mathfrak{h}}^{(r)}(f)$ as an $\mathcal{O}$-module as explained above.

\section{Higher PRIMItive IDEALS}

In this section we will discuss a relative notion associated with derivations and idealizers.

Definition 3. (i) Let $\mathfrak{h} \subset \mathfrak{g} \subset \mathcal{O}$ be ideals, and let $r \geq 0$ be an integer. The rth primitive ideal of $\mathfrak{g}$ (relative to $\mathfrak{h}$ ) is

$$
\oint_{\mathfrak{h}}^{(r)} \mathfrak{g}:=\left\{f \in \mathfrak{g} \mid \mathbb{I}_{\mathfrak{h}}^{(r)}(f) \subset \mathfrak{g}\right\}
$$

(ii) The rth iterated primitive ideal of $\mathfrak{g}$ relative to $\mathfrak{h}$ is

$$
\int_{\mathfrak{h}}^{(r)} \mathfrak{g}:=\left\{f \in \mathfrak{g} \mid\left(\mathbb{I}_{\mathfrak{h}}\right)^{r}(f) \subset \mathfrak{g}\right\} .
$$

Clearly, $\oint_{\mathfrak{h}}^{(r)} \mathfrak{g} \subset \int_{\mathfrak{h}}^{(r)} \mathfrak{g}$ and, trivially, $\oint_{\mathfrak{h}}^{(0)} \mathfrak{g}=\int_{\mathfrak{h}}^{(0)} \mathfrak{g}=\mathfrak{g}$. If $r=1$, we omit the superscript, setting $\oint_{\mathfrak{h}}^{(1)} \mathfrak{g}=\int_{\mathfrak{h}}^{(1)} \mathfrak{g}=\int_{\mathfrak{h}} g$. Note that for this value of $r$ the present definition coincides with that introduced by Pellikaan (cf. [6], 7], also [2]). Fixing the ideal $\mathfrak{h}$, the question as to when one has equalities $\oint_{\mathfrak{h}}^{(r)} \mathfrak{g}=\int_{\mathfrak{h}}^{(r)} \mathfrak{g}$ throughout for all ideals $\mathfrak{g} \supset \mathfrak{h}$ and for all values of $r$ is rather subtle. We suspect it may be very close to implying that $\mathcal{O} / \mathfrak{h}$ be a regular ring. The question is also related to the well known conjecture of Nakai on differential operators.

As it turns out, the first notion is rather unwielding in several aspects, theoretical as well as computational. The clear advantage of the second notion is that it is recursively defined, according to the following result.

Proposition 4. Let $\mathfrak{h} \subset \mathfrak{g} \subset \mathcal{O}$ be ideals. Then $\int_{\mathfrak{h}}^{(r)} \mathfrak{g}=\left\{f \in \mathfrak{g} \mid \mathbb{I}_{\mathfrak{h}}(f) \subset \int_{\mathfrak{h}}^{(r-1)} \mathfrak{g}\right\}$. In particular, $\int_{\mathfrak{h}}^{(r)} \mathfrak{g}=\int_{\mathfrak{h}}\left(\int_{\mathfrak{h}}^{(r-1)} \mathfrak{g}\right)$.

Proof. Set iteratively, for $r \geq 0$,

$$
\int_{\mathfrak{h}}^{((r-1 \rightarrow r))} \mathfrak{g}:=\left\{f \in \mathfrak{g} \mid \mathbb{I}_{\mathfrak{h}}(f) \subset \int_{\mathfrak{h}}^{((r-2 \rightarrow r-1))} \mathfrak{g}\right\}
$$

with the initial condition $\int_{\mathfrak{h}}^{((-1 \rightarrow 0))} \mathfrak{g}:=\mathfrak{g}$.

We will show that $\int_{\mathfrak{h}}^{(r)} \mathfrak{g}=\int_{\mathfrak{h}}^{((r-1 \rightarrow r))} \mathfrak{g}$, for every integer $r \geq 0$. This easily implies our contention. To prove the latter result, we argue inductively. Thus, let $r \geq 0$. Since $\int_{\mathfrak{h}}^{(0)} \mathfrak{g}=\int_{\mathfrak{h}}^{((-1 \rightarrow 0))} \mathfrak{g}(=\mathfrak{g})$, we assume inductively that $\int_{\mathfrak{h}}^{(s)} \mathfrak{g}=$ $\int_{\mathfrak{h}}^{((s-1 \rightarrow s))} \mathfrak{g}$ for $s \leq r-1$. We show the inclusion $\int_{\mathfrak{h}}^{(r)} \mathfrak{g} \subset \int_{\mathfrak{h}}^{((r-1 \rightarrow r))} \mathfrak{g}$, the opposite one being entirely similar. Thus, let $f \in \int_{\mathfrak{h}}^{(r)} \mathfrak{g}$ and let $\delta \in \mathbb{I}_{\mathfrak{h}}(f)$ be arbitrary. We wish to show that $\delta(f) \in \int_{\mathfrak{h}}^{((r-2 \rightarrow r-1))} \mathfrak{g}$. By the inductive hypothesis, $\int_{\mathfrak{h}}^{(r-1)} \mathfrak{g}=\int_{\mathfrak{h}}^{((r-2 \rightarrow r-1))} \mathfrak{g}$; hence we have to show that $\delta(f) \in \int_{\mathfrak{h}}^{(r-1)} \mathfrak{g}$, i.e., that 
$\left(\delta_{1} \cdots \delta_{t}\right)(\delta(f)) \in \mathfrak{g}$, for all $\delta_{1} \cdots \delta_{t} \in \mathbb{I}_{\mathfrak{h}}(f)$ and for every $t \leq r-1$. However, $\left(\delta_{1} \cdots \delta_{t}\right)(\delta(f))=\left(\delta_{1} \cdots \delta_{t} \cdot \delta\right)(f) \in \mathfrak{g}$ since $f \in \int_{\mathfrak{h}}^{(r)} \mathfrak{g}$ by assumption and since we have a composite of $t+1 \leq r$ derivations of order one.

Remark 5. It is easy to see that in both versions of the primitive ideal one can replace the respective forms of the differential idealizer by their essential parts, without affecting the result. This technical point will often simplify the argument of a proof.

This seemingly innocuous result allows us to deduce rather quickly a few important results. Recall that for any commutative noetherian ring $A$ and any ideal $I \subset A$, we have the notion of the $s$ th symbolic power $I^{(s)}$ of $I$, where $s$ is a nonnegative integer. Namely, $I^{(s)}$ is the intersection of the primary components of $I^{s}$ relative to the minimal primes of $I$. Equivalently, $I^{(s)}=\{a \in A \mid \exists x \in$ $A \backslash \bigcup_{P \in \operatorname{Min}(A / I)} P$, with $\left.x a \in I^{s}\right\}$.

We now return to our original settings. From now on, most of our results will refer to the iterated primitive ideal.

Proposition 6. Let $\mathcal{O}$ be a commutative noetherian $k$-algebra and let $\mathfrak{h} \subset \mathfrak{g} \subset \mathcal{O}$ be ideals. Then:

(i) For every $r \geq 0$, the annihilator of the $\mathcal{O}$-module $\int_{\mathfrak{h}}^{(r)} \mathfrak{g} / \int_{\mathfrak{h}}^{(r+1)} \mathfrak{g}$ contains $\mathfrak{g}$.

(ii) If $\mathfrak{g}$ has no embedded associated primes, then $\operatorname{Ass}(\mathcal{O} / \mathfrak{g})=\operatorname{Ass}\left(\mathcal{O} / \int_{\mathfrak{h}}^{(r)} \mathfrak{g}\right)$ for every $r \geq 0$.

(iii) If $\mathfrak{g}$ has no embedded associated primes, then, for every $r \geq 0, \mathfrak{g}^{(r+1)} \subset$ $\widetilde{\mathfrak{g}^{(r+1)}} \subset \int_{\mathfrak{h}}^{(r)} \mathfrak{g}$, where $\widetilde{\mathfrak{g}^{(r+1)}}$ denotes the inverse image in $\mathcal{O}$ of $(\mathfrak{g} / \mathfrak{h})^{(r+1)} \subset$ $\mathcal{O} / \mathfrak{h}$. Moreover, $\operatorname{Ass}(\mathcal{O} / \mathfrak{g})=\operatorname{Ass}\left(\mathcal{O} / \widetilde{\mathfrak{g}^{(r+1)}}\right)$.

(iv) The sequence $\left\{\int_{\mathfrak{h}}^{(r)} \mathfrak{g}\right\}_{r \geq 0}$ is a descending multiplicative filtration.

Proof. (i) Proceed by induction on $r$. For $r=0$, we must verify that $\mathfrak{g}^{2} \subset \int_{\mathfrak{h}} \mathfrak{g}$. As will be seen shortly, this part is already subsumed in the general step of the recursion. Thus, let $f \in \int_{\mathfrak{h}}^{(r)} \mathfrak{g}$ and $g \in \mathfrak{g}$. Let $\delta \in \mathbb{I}_{\mathfrak{h}}$. Then $\delta(g f)=\delta(g) f+g \delta(f)$. The first summand on the right-hand side belongs to $\int_{\mathfrak{h}}^{(r)} \mathfrak{g}$ (since $f$ does). Since $\delta(f) \in \int_{\mathfrak{h}}^{(r-1)} \mathfrak{g}$ by assumption, the second summand belongs to $\mathfrak{g} \int_{\mathfrak{h}}^{(r-1)} \mathfrak{g}$. The latter is contained in $\int_{\mathfrak{h}}^{(r)} \mathfrak{g}$ by the inductive hypothesis. Thus, we have shown that $\delta(g f) \in \int_{\mathfrak{h}}^{(r)} \mathfrak{g} ;$ hence $g f \in \int_{\mathfrak{h}}^{(r+1)} \mathfrak{g}$, as required.

(ii) By part (i) and induction on $r$, one has $\mathfrak{g}^{r+1} \subset \int_{\mathfrak{h}}^{(r)} \mathfrak{g}(\subset \mathfrak{g})$ for every $r \geq 0$. Therefore, $\int_{\mathfrak{h}}^{(r)} \mathfrak{g}$ and $\mathfrak{g}$ have the same radical for every $r \geq 0$. On the other hand, it follows immediately from the definitions that, for any two ideals $\mathfrak{g}_{1}, \mathfrak{g}_{2}$ containing $\mathfrak{h}, \int_{\mathfrak{h}}^{(r)}\left(\mathfrak{g}_{1} \cap \mathfrak{g}_{2}\right)=\int_{\mathfrak{h}}^{(r)} \mathfrak{g}_{1} \cap \int_{\mathfrak{h}}^{(r)} \mathfrak{g}_{2}$. Since $\mathfrak{g}$ has no embedded primes by assumption, one may therefore stick to the case where $\mathfrak{g}$ is a primary ideal.

Say $\{P\}=\operatorname{Ass}(\mathcal{O} / \mathfrak{g})$. From the exact sequence

$$
0 \longrightarrow \mathfrak{g} / \int_{\mathfrak{h}}^{(r)} \mathfrak{g} \longrightarrow \mathcal{O} / \int_{\mathfrak{h}}^{(r)} \mathfrak{g} \longrightarrow \mathcal{O} / \mathfrak{g} \longrightarrow 0
$$


one has $\operatorname{Ass}\left(\mathcal{O} / \int_{\mathfrak{h}}^{(r)} \mathfrak{g}\right) \subset \operatorname{Ass}\left(\mathfrak{g} / \int_{\mathfrak{h}}^{(r)} \mathfrak{g}\right) \cup \operatorname{Ass}(\mathcal{O} / \mathfrak{g})$. Therefore, it suffices to show that $\mathfrak{g} / \int_{\mathfrak{h}}^{(r)} \mathfrak{g}$ is $P$-primary. For that, assume that $Q \in \operatorname{Spec}(\mathcal{O})$ is an associated prime of the latter module, say, $Q=\operatorname{ann}_{\mathcal{O}}(\bar{x})$, for some $x \in \mathfrak{g} \backslash \int_{\mathfrak{h}}^{(r)} \mathfrak{g}$. It suffices to show that $Q \subset P$. Letting $a \in Q$, we have $a x \in \int_{\mathfrak{h}}^{(r)} \mathfrak{g}$. By definition, for any $\xi \in\left(\mathbb{I}_{\mathfrak{h}}\right)^{r}, \xi(a x) \equiv a \xi(x) \equiv 0(\bmod \mathfrak{g})$. If $a \notin P$, we must have $\xi(x) \in \mathfrak{g}$. But then $x \in \int_{\mathfrak{h}}^{(r)} \mathfrak{g}$, leading to a contradiction.

(iii) The inclusion $\mathfrak{g}^{(r+1)} \subset \widetilde{\mathfrak{g}^{(r+1)}}$ follows trivially from the definition of the symbolic powers, so we only have to provide the second inclusion of the chain. We proceed by induction on $r$, the result being trivial for $r=0$. For this, let $f \in \overline{\mathfrak{g}^{(r+1)}}$. By Proposition 4 we will show that $\delta(f) \in \int_{\mathfrak{h}}^{(r-1)} \mathfrak{g}$ for every $\delta \in \mathbb{I}_{\mathfrak{h}}$. By definition of the symbolic power, there exists $x \in \mathcal{O} \backslash \mathcal{Z}((\mathcal{O} / \mathfrak{h}) /(\mathfrak{g} / \mathfrak{h}))=\mathcal{O} \backslash \mathcal{Z}(\mathcal{O} / \mathfrak{g})$ such that $x f \in \mathfrak{g}^{r+1}+\mathfrak{h}$. By part $(\mathrm{i}), \mathfrak{g}^{r+1} \subset \int_{\mathfrak{h}}^{(r)} \mathfrak{g}$. Since $\mathfrak{h} \subset \int_{\mathfrak{h}}^{(r)} \mathfrak{g}$ trivially then $x f \in \int_{\mathfrak{h}}^{(r)} \mathfrak{g}$. It follows that $\delta(x) f+x \delta(f)=\delta(x f) \in \int_{\mathfrak{h}}^{(r-1)} \mathfrak{g}$. But $f \in \widetilde{\mathfrak{g}^{(r+1)}} \subset \widetilde{\mathfrak{g}^{(r)}}$ (the latter because of the evident inclusion of successive descending symbolic powers in general). By the inductive hypothesis, $\widetilde{\mathfrak{g}^{(r)}} \subset \int_{\mathfrak{h}}^{(r-1)} \mathfrak{g}$. Then also $x \delta(f) \in \int_{\mathfrak{h}}^{(r-1)} \mathfrak{g}$. By the assumption on $x$ and by part (ii), we must have $\delta(f) \in \int_{\mathfrak{h}}^{(r-1)} \mathfrak{g}$, as was to be shown.

As for the statement about the associated primes, one notes that the associated primes of $\mathfrak{g} / \mathfrak{h}$ come from those of $\mathfrak{g}$; hence $\mathfrak{g} / \mathfrak{h}$ has no embedded primes as well. In this case, Ass $(\mathfrak{g} / \mathfrak{h})=\operatorname{Ass}\left((\mathfrak{g} / \mathfrak{h})^{(r+1)}\right)$ for any $r \geq 1$ by the definition of symbolic powers. Since the associated primes of $\widetilde{\mathfrak{g}^{(r+1)}}$ are the inverse images of those of $(\mathfrak{g} / \mathfrak{h})^{(r+1)}$, the assertion follows.

(iv) By definition, the sequence is a descending filtration. To see that it is moreover multiplicative, let $r_{i} \geq 0$ and let $f_{i} \in \int_{\mathfrak{h}}^{\left(r_{i}\right)} \mathfrak{g}(i=1,2)$. Proceed by induction on the sum $r_{1}+r_{2}$, the result being trivial if $r_{1}+r_{2}=0$. Thus, let $r_{1}+r_{2} \geq 1$. Let $\delta \in \mathbb{I}_{\mathfrak{h}}$. Then

$$
\begin{aligned}
\delta\left(f_{1} f_{2}\right) & =\delta\left(f_{1}\right) f_{2}+f_{1} \delta\left(f_{2}\right) \\
& \in \int_{\mathfrak{h}}^{\left(r_{1}-1\right)} \mathfrak{g} \cdot \int_{\mathfrak{h}}^{\left(r_{2}\right)} \mathfrak{g}+\int_{\mathfrak{h}}^{\left(r_{1}\right)} \mathfrak{g} \cdot \int_{\mathfrak{h}}^{\left(r_{2}-1\right)} \mathfrak{g} \subset \int_{\mathfrak{h}}^{\left(r_{1}+r_{2}-1\right)} \mathfrak{g},
\end{aligned}
$$

the last inclusion following from the inductive hypothesis. The required result now follows from Proposition 4

We next need the following technical lemma, which was essentially obtained in [9. (1.3) Lemma]. Since we actually prove a bit more, a proof is included for completeness.

Lemma 7. Let $\mathcal{O}$ be a $k$-algebra of finite type over a commutative ring $k$. Let $\mathfrak{h} \subset \mathfrak{g} \subset \mathcal{O}$ be ideals having no embedded primes and let $\mathcal{S} \subset \mathcal{O}$ be a multiplicative set. Then $\mathcal{S}^{-1}\left(\int_{\mathfrak{h}}^{(r)} \mathfrak{g}\right) \simeq \int_{\mathcal{S}^{-1} \mathfrak{h}}^{(r)} \mathcal{S}^{-1} \mathfrak{g}$ for every $r \geq 0$.

Proof. We first show that $\mathcal{S}^{-1} \mathbb{I}_{\mathfrak{h}} \simeq \mathbb{I}_{\mathcal{S}^{-1} \mathfrak{h}}$ in a natural way. If $\mathcal{S}$ meets some minimal prime of $\mathfrak{h}$, then $\mathcal{S}^{-1} \mathfrak{h}=\mathcal{S}^{-1} \mathcal{O}$; hence clearly $\mathbb{I}_{\mathcal{S}^{-1} \mathfrak{h}}=\operatorname{Diff}^{(1)}\left(\mathcal{S}^{-1} \mathcal{O}\right)$, while $\mathcal{S}^{-1} \mathbb{I}_{\mathfrak{h}}=$ $\operatorname{Diff}^{(1)}\left(\mathcal{S}^{-1} \mathcal{O}\right)$ since $\mathbb{I}_{\mathfrak{h}} \supset \mathfrak{h} \operatorname{Difff}^{(1)}(\mathcal{O})$. 
Thus, assume that $\mathcal{S}$ does not meet any minimal prime of $\mathfrak{h}$. There is a natural isomorphism $\mathcal{S}^{-1} \operatorname{Diff}^{(1)}(\mathcal{O}) \stackrel{\varphi}{\simeq} \operatorname{Diff}^{(1)}\left(\mathcal{S}^{-1} \mathcal{O}\right)$ (cf. [1]). Precisely, if $t^{-1} \delta \in$ $\mathcal{S}^{-1} \operatorname{Diff}^{(1)}(\mathcal{O})$ and if $s^{-1} f \in \mathcal{S}^{-1} \mathcal{O}$, then $\left(\varphi\left(t^{-1} \delta\right)\right)\left(s^{-1} f\right)=\left(t s^{2}\right)^{-1}(\delta(s) f-s \delta(f))$. It is pretty obvious then that the restriction of $\varphi$ to $\mathcal{S}^{-1} \mathbb{I}_{\mathfrak{h}}$ maps (injectively) to $\mathbb{I}_{\mathcal{S}^{-1} \mathfrak{h}}$. It remains to show that it is onto this module. For that, let $\xi \in \mathbb{I}_{\mathcal{S}^{-1} \mathfrak{h}}$ and let $t^{-1} \delta=\varphi^{-1}(\xi) \in \mathcal{S}^{-1} \operatorname{Diff}^{(1)}(\mathcal{O})$. Let $f \in \mathfrak{h}$. Then $\xi(f / 1)=\left(\varphi\left(t^{-1} \delta\right)\right)(f / 1)=$ $(t)^{-1}(\delta(1) f-\delta(f)) \in \mathcal{S}^{-1} \mathfrak{h}$ by assumption; hence $\delta(f) / 1 \in \mathcal{S}^{-1} \mathfrak{h}$, so $s \delta(f) \in \mathfrak{h}$ for some $s \in \mathcal{S}$. But $s$ is not a zero-divisor on $\mathfrak{h}$ by hypothesis. Therefore, $\delta(f) \in \mathfrak{h}$, thus showing that $t^{-1} \delta \in \mathcal{S}^{-1} \mathbb{I}_{\mathfrak{h}}$, as intended.

The same sort of argument shows that the restriction of $\varphi$ to $\mathcal{S}^{-1} \widehat{\mathbb{I}_{\mathfrak{h}}}$, where $\widehat{\mathbb{I}_{\mathfrak{h}}}$ is the essential part of $\mathbb{I}_{\mathfrak{h}}$, maps isomorphically onto the essential part $\overline{\mathbb{I}_{\mathcal{S}^{-1} \mathfrak{h}}}$.

We now prove the main assertion. We induct on $r$, starting with $r=0$. Here there is nothing to prove since $\mathcal{S}^{-1} \int_{\mathfrak{h}}^{(0)} \mathfrak{g}=\mathcal{S}^{-1} \mathfrak{g}=\int_{\mathcal{S}^{-1} \mathfrak{h}}^{(0)} \mathcal{S}^{-1} \mathfrak{g}$. Now, assume the result to be true for $r-1$. By Proposition 4 and Remark [5, one has

$$
\int_{\mathfrak{h}}^{(r)} \mathfrak{g}=\left\{g \in \mathfrak{g} \mid \widehat{\mathbb{I}_{\mathfrak{h}}}(g) \subset \int_{\mathfrak{h}}^{(r-1)} \mathfrak{g}\right\},
$$

and, similarly,

$$
\int_{\mathcal{S}^{-1} \mathfrak{h}}^{(r)} \mathcal{S}^{-1} \mathfrak{g}=\left\{g \in \mathfrak{g} \mid \widehat{\mathbb{I}_{\mathcal{S}^{-1} \mathfrak{h}}}(g) \subset \int_{\mathcal{S}^{-1} \mathfrak{h}}^{(r-1)} \mathcal{S}^{-1} \mathfrak{g}\right\}
$$

By the first part of the proof, $\mathcal{S}^{-1} \widehat{\mathbb{I}_{\mathfrak{h}}} \simeq \widehat{\mathbb{I}_{\mathcal{S}^{-1} \mathfrak{h}}}$ via $\varphi$ and, by the inductive hypothesis, $\mathcal{S}^{-1} \int_{\mathfrak{h}}^{(r-1)} \mathfrak{g}=\int_{\mathcal{S}^{-1} \mathfrak{h}}^{(r-1)} \mathcal{S}^{-1} \mathfrak{g}$.

First, let $g \in \int_{\mathfrak{h}}^{(r)} \mathfrak{g}$ and $\eta \in \widehat{\mathbb{I}_{\mathcal{S}^{-1} \mathfrak{h}}}=\varphi\left(\mathcal{S}^{-1} \widehat{\mathbb{I}_{\mathfrak{h}}}\right)$. Say $\eta=\varphi\left(t^{-1} \delta\right)$ with $\delta \in \mathbb{I}_{\mathfrak{h}}$. Applying $\eta$ to $g / 1$, we find $t^{-1}(\delta(1) g-\delta(g))=-t^{-1} \delta(g)$ (here, use $\delta(1)=0$ since $\delta$ is an ordinary derivation of $\mathcal{O})$. The latter element belongs to $\mathcal{S}^{-1} \int_{\mathfrak{h}}^{(r-1)} \mathfrak{g}=$ $\int_{\mathcal{S}^{-1} \mathfrak{h}}^{(r-1)} \mathcal{S}^{-1} \mathfrak{g}$ as $\delta(g) \in \int_{\mathfrak{h}}^{(r-1)} \mathfrak{g}$ by hypothesis. This shows that the image of $\int_{\mathfrak{h}}^{(r)} \mathfrak{g}$ by the canonical homomorphism $\mathcal{O} \rightarrow \mathcal{S}^{-1} \mathcal{O}$ is a subideal of $\int_{\mathcal{S}^{-1} \mathfrak{h}}^{(r)} \mathcal{S}^{-1} \mathfrak{g}$, which implies the inclusion $\mathcal{S}^{-1} \int_{\mathfrak{h}}^{(r)} \mathfrak{g} \subset \int_{\mathcal{S}^{-1} \mathfrak{h}}^{(r)} \mathcal{S}^{-1} \mathfrak{g}$.

Conversely, let $s^{-1} g \in \int_{\mathcal{S}^{-1} \mathfrak{h}}^{(r)} \mathcal{S}^{-1} \mathfrak{g}$. For the purpose of proving the required reverse inclusion, we may assume that $s=1$. Let $\delta \in \widehat{\mathbb{I}_{\mathfrak{h}}}$. Then $\varphi(\delta / 1) \in \varphi\left(\mathcal{S}^{-1} \widehat{\mathbb{I}_{\mathfrak{h}}}\right)=$ $\widehat{\mathbb{I}_{\mathcal{S}^{-1} \mathfrak{h}}}$; hence by assumption, $\varphi(\delta / 1)(g / 1) \in \int_{\mathcal{S}^{-1} \mathfrak{h}}^{(r-1)} \mathcal{S}^{-1} \mathfrak{g}=\mathcal{S}^{-1} \int_{\mathfrak{h}}^{(r-1)} \mathfrak{g}$. Again, $\varphi(\delta / 1)(g / 1)=-\delta(g) / 1$ (since $\delta(1)=0)$. Therefore, $t \delta(g) \in \int_{\mathfrak{h}}^{(r-1)} \mathfrak{g}$, for some $t \in \mathcal{S}$.

Since the notion of primitive ideal clearly commutes with taking intersections at the level of $\mathfrak{g}$ (cf. proof of Proposition 6] (ii)), and so does taking fractions, we may assume that $\mathfrak{g}$ is primary. Say $P$ is the radical of $\mathfrak{g}$. Moreover, we may also assume that $P \cap \mathcal{S}=\emptyset$; otherwise there is nothing to prove, as $\int_{\mathfrak{h}}^{(r)} \mathfrak{g}$ is again $P$-primary by Proposition 6, (ii). It follows that $\delta(g) \in \int_{\mathfrak{h}}^{(r-1)} \mathfrak{g}$, as was required to show.

One can give some conditions under which the iterated primitive ideal coincides with the inverse image of the relative symbolic power (phased out by one). Assume that $k$ is a field and that $\mathcal{O}$ is a polynomial ring over $k$. We will next state a result in terms of algebraic conditions. 
Recall that, for an affine ring $\mathcal{O} / \mathfrak{h}$, with $\mathfrak{h}=\left(f_{1}, \ldots, f_{m}\right)$, its Jacobian ideal is the ideal $(\mathfrak{J}(\mathfrak{h})+\mathfrak{h}) / \mathfrak{h}$, where $\mathfrak{J}(\mathfrak{h}) \subset \mathcal{O}$ is the ideal generated by the $c \times c$ minors of the Jacobian matrix of $\left\{f_{1}, \ldots, f_{m}\right\}$ with $c$ standing for the codimension of $\mathfrak{h}$. (This is a Fitting ideal; hence it is independent of the choice of generators of $\mathfrak{h}$ ).

Proposition 8. Let $\mathcal{O}=k\left[z_{1}, \ldots, z_{n}\right]$ be a polynomial ring over a field $k$ of characteristic zero and let $\mathfrak{h} \subset \mathfrak{g} \subset \mathcal{O}$ be ideals, with $\mathfrak{h}$ unmixed and $\mathfrak{g}$ radical. If $\mathfrak{J}(\mathfrak{h})$ is not contained in any minimal prime of $\mathcal{O} / \mathfrak{g}$, then $\int_{\mathfrak{h}}^{(r)} \mathfrak{g}=\widetilde{\mathfrak{g}^{(r+1)}}$ for every $r \geq 0$.

Proof. By Proposition [6 neither $\int_{\mathfrak{h}}^{(r)} \mathfrak{g}$ nor $\widetilde{\mathfrak{g}^{(r+1)}}$ has embedded primes and their common radical is $\mathfrak{g}$. Therefore, it suffices to show that the two ideals are equal locally at the associated primes of $\mathfrak{g}$.

By using the isomorphism of $\mathcal{O} / \mathfrak{h}$-modules $\operatorname{Diff}^{1}(\mathcal{O} / \mathfrak{h}) \simeq \mathbb{I}_{\mathfrak{h}} / \mathfrak{h} \operatorname{Diff}^{1}(\mathcal{O})(\mathrm{cf}$. [4]), it follows from the recursive character of the iterated primitive ideal that $\left(\int_{\mathfrak{h}}^{(r)} \mathfrak{g}\right) / \mathfrak{h}=$ $\int_{0}^{(r)}(\mathfrak{g} / \mathfrak{h})$. Then, by Lemma 7, we have $\left(\int_{\mathfrak{h}_{\wp}}^{(r)} \mathfrak{g}_{\wp}\right) / \mathfrak{h}_{\wp}=\int_{0}^{(r)}(\mathfrak{g} / \mathfrak{h})_{\wp / \mathfrak{h}}$. Since both sides commute with intersections and $\mathfrak{g}$ is assumed to be a radical ideal, we may suppose that $\mathfrak{g}$ is a prime ideal. The hypothesis that $\mathfrak{J}(\mathfrak{h})$ is not contained in $\mathfrak{g}$ implies that $\mathfrak{g}$ belongs to the regular locus of $\mathcal{O} / \mathfrak{h}$. In this case, by [9, (3.2) Theorem], we have $\int_{0}^{(r)}(\mathfrak{g} / \mathfrak{h})_{\wp / \mathfrak{h}}=(\mathfrak{g} / \mathfrak{h})_{\wp / \mathfrak{h}}^{(r+1)}$. This yields our contention.

Remark 9. We suspect that a version of Proposition 8 remains valid if $\mathfrak{g}$ is a (not necessarily radical) complete intersection locally on primes in $V(\mathfrak{h}) \backslash V(\mathfrak{J}(\mathfrak{h}))$. In general, the requirement that $(\mathfrak{g}: \mathfrak{J}(\mathfrak{h}))=\mathfrak{g}$ is needed for the result to hold. For example, let $\mathfrak{g}$ be generated by variables, say, $\mathfrak{g}=\left(z_{1}, \ldots, z_{k}\right)(k \leq n)$ and let $\mathfrak{h} \subset \mathfrak{g}^{2}$ be generated by the monomials of degree 2 in $z_{1}, \ldots, z_{c}(c \leq k)$. A calculation shows that $\int_{\mathfrak{h}} \mathfrak{g}=\left(z_{1}, \ldots, z_{c}\right)+\left(z_{c+1}, \ldots, z_{k}\right)^{2}$. Nevertheless, as one easily sees, $\widetilde{\mathfrak{g}^{(2)}}=\mathfrak{g}^{2}$. Of course, the condition $\mathfrak{h} \subset \mathfrak{g}^{2}$ implies that $\mathfrak{J}(\mathfrak{h}) \subset \mathfrak{g}$.

\section{The COMPUtATION OF THE PRIMITIVE IDEAL}

In this section we show how to effectively compute the $r$ th iterated primitive ideal in case $\mathcal{O}$ is a polynomial ring over a field $k$ of characteristic zero. The basic computational result refers to the first primitive ideal. We make the convention of writing the elements of the $\mathcal{O}$-submodule $\widehat{\mathbb{I}_{\mathfrak{h}}} \subset \operatorname{Der}(\mathcal{O})=\sum_{i=1}^{n} \mathcal{O} \partial / \partial z_{i}$ as column vectors with coordinates in $\mathcal{O}$.

Proposition 10. Let $\mathfrak{h} \subset \mathfrak{g} \subset \mathcal{O}$ be ideals. Let $\mathfrak{g}=(\mathrm{g})=\left(g_{1}, \ldots, g_{m}\right)$ be generators of $\mathfrak{g}$, let $\Theta(\mathbf{g})$ denote the Jacobian matrix of $\mathfrak{g}$ and let $\Delta(\mathfrak{h})$ denote the matrix whose columns are a set of generators of the $\mathcal{O}$-submodule $\widehat{\mathbb{I}}_{\mathfrak{h}} \subset \operatorname{Der}(\mathcal{O})$. Then

$$
\int_{\mathfrak{h}} \mathfrak{g}=I_{1}(\mathfrak{g} \cdot \Psi)
$$

where $\Psi$ denotes the lifting to $\mathcal{O}$ of the syzygy matrix of the transpose of $\Theta(\mathrm{g}) \cdot \Delta(\mathfrak{h})$ modulo the ideal $\mathfrak{g}$.

Proof. Let $g=\sum_{j} u_{j} g_{j} \in \mathfrak{g}$ and let $\delta=\sum_{i} h_{i} \partial / \partial z_{i} \in \widehat{\mathbb{I}_{\mathfrak{h}}}$. Then

$$
\delta(g)=\left(\sum_{i} h_{i} \partial / \partial z_{i}\right)\left(\sum_{j} u_{j} g_{j}\right)=\sum_{j} u_{j}\left(\sum_{i} h_{i} \partial g_{j} / \partial z_{i}\right)+\sum_{j} g_{j}\left(\sum_{i} h_{i} \partial u_{j} / \partial z_{i}\right) ;
$$


hence $\delta(g) \in \mathfrak{g}$ if and only if $\sum_{j} u_{j}\left(\sum_{i} h_{i} \partial g_{j} / \partial z_{i}\right) \in \mathfrak{g}$. Letting $\delta$ run through a set of generators $\left\{\sum_{i} h_{i k} \partial / \partial z_{i}\right\}_{1 \leq k \leq p}$ of $\widehat{\mathbb{I}_{\mathfrak{h}}}$, this condition means that the entries of the product

$$
\left(\begin{array}{cccc}
\sum_{i} h_{i 1} \partial g_{1} / \partial z_{i} & \sum_{i} h_{i 1} \partial g_{2} / \partial z_{i} & \ldots & \sum_{i} h_{i 1} \partial g_{m} / \partial z_{i} \\
\vdots & \vdots & \vdots & \vdots \\
\sum_{i} h_{i p} \partial g_{1} / \partial z_{i} & \sum_{i} h_{i p} \partial g_{2} / \partial z_{i} & \ldots & \sum_{i} h_{i p} \partial g_{m} / \partial z_{i}
\end{array}\right) \cdot\left(\begin{array}{c}
u_{1} \\
u_{2} \\
\vdots \\
u_{m}
\end{array}\right)
$$

belong to $\mathfrak{g}$. But the transpose of the matrix on the left is the product $\Theta(\mathbf{g}) \cdot \Delta(\mathfrak{h})$. Therefore, $\delta(g) \in \mathfrak{g}$ for every $\delta \in \widehat{\mathbb{I}_{\mathfrak{h}}}$ if and only if the column vector on the right is a syzygy of this matrix modulo $\mathfrak{g}$.

Remark 11. The previous result, along with Proposition 4 yields an effective way of computing iterated primitive ideals. By shifting emphasis, the result can be viewed as a means of effectively computing symbolic powers of an ideal in a residue ring of a polynomial ring in a simple recursive way (that avoids, say, taking the unmixed part of the ordinary powers by means of computing with Ext modules and resolutions).

\section{Applications}

A couple of examples may indicate the applications of higher relative primitive ideals. Let $X \supset Y$ be germs of reduced analytic subspaces in $\left(\mathbb{C}^{n}, 0\right)$, defined by radical ideals $\mathfrak{h} \subset \mathfrak{g} \subset \mathcal{O}$. The higher iterated primitive ideals roughly tell us how $Y$ is embedded in $X$. The case when $X$ is smooth was considered by Pellikaan 6]. In case $X$ is singular and $Y$ is a smooth curve, the (first) primitive ideal was computed in [2].

Also when $X$ is a two dimensional isolated singular hypersurface, the length $\lambda$ of the torsion submodule $T$ of the conormal module of $\mathfrak{g} / \mathfrak{h}$ was used as a relative invariant of $Y$ with respect to $X$ [3]. It was proved that $T=\left(\int_{h}^{(1)} \mathfrak{g}\right) / \mathfrak{g}^{2}+\mathfrak{h}$. It is quite conceivable that $\lambda$ remains unchanged for different smooth curves on the same surface $X$ with an isolated singularity.

Example 1. Let $\mathfrak{h}=x y+(1 / k+1) z^{k+1}$ be the well known $A_{k}$ singularity and let $\mathfrak{g}=(y, z)$ be a line contained therein. Then

$$
\int_{\mathfrak{h}}^{(r)} \mathfrak{g}= \begin{cases}\left(y, z^{r+1}\right) & \text { if } r \leq k \\ y \int_{\mathfrak{h}}^{(r-k-1)} \mathfrak{g}+\mathfrak{h} & \text { otherwise. }\end{cases}
$$

Example 2. In the first example the line in consideration had $\lambda=1$. If we take a line with $\lambda=l$ on an $A_{2 l-1}$ surface to be the $x$-axis, then $X$ is defined by $\mathfrak{h}_{l}=x^{l} y+z^{2}+y z($ see [3] $)$. In this case we have

$$
\int_{\left(h_{l}\right)}^{(r)} \mathfrak{g}= \begin{cases}y^{\frac{r}{2}}(y, z)+\left(h_{l}\right) & \text { if } r=2 k \\ y^{\frac{r-1}{2}}\left(y, z^{2}\right)+\left(h_{l}\right) & \text { if } r=2 k+1 .\end{cases}
$$

Example 3. Consider the $D_{5}$ surface $D_{5,2}$, with defining equation $h_{1}=x^{2} y+y^{4}+$ $z^{2}$ with respect to the line defined by the ideal $\mathfrak{g}=(y, z)$. Then

$$
\int_{\left(h_{1}\right)}^{(1)} \mathfrak{g}=\left(y, z^{2}\right), \quad \int_{\left(h_{1}\right)}^{(2)} \mathfrak{g}=y \mathfrak{g}+\mathfrak{h}_{1}, \quad \int_{\left(h_{1}\right)}^{(r+1)} \mathfrak{g}=\left(y^{r}\right)+\mathfrak{h}_{1}, \quad(r>2) .
$$


Example 4. Consider a $D_{5}$ surface once more, only now with respect to a different line being the $x$-axis. The equation of the surface is $D_{5,2}^{*}: h_{2}=x^{2} y+x z^{2}+y^{2}$. Here we obtain

$$
\int_{\left(h_{2}\right)}^{(1)} \mathfrak{g}=\left(y, z^{2}\right), \quad \int_{\left(h_{2}\right)}^{(2)} \mathfrak{g}=\left(y z, x y+z^{2}\right)+\left(h_{2}\right), \quad \int_{\left(h_{2}\right)}^{(3)} \mathfrak{g}=\left(y z^{2}, x y+z^{2}\right)+\left(h_{2}\right),
$$

and, for $s \geq 1$,

$$
\begin{gathered}
\int_{\left(h_{2}\right)}^{(4 s)} \mathfrak{g}=\left(x y+z^{2}\right)^{s} \mathfrak{g}+\left(h_{2}\right), \quad \int_{\left(h_{2}\right)}^{(4 s+1)} \mathfrak{g}=\left(x y+z^{2}\right)^{s} \int_{h_{2}}^{(1)} \mathfrak{g}+\left(h_{2}\right), \\
\int_{\left(h_{2}\right)}^{(4 s+2)} \mathfrak{g}=\left(x y+z^{2}\right)^{s} \int_{h_{2}}^{(2)} \mathfrak{g}+\left(h_{2}\right), \quad \int_{\left(h_{2}\right)}^{(4 s+3)} \mathfrak{g}=\left(x y+z^{2}\right)^{s} \int_{h_{2}}^{(3)} \mathfrak{g}+\left(h_{2}\right) .
\end{gathered}
$$

We note that the invariant $\lambda=2$ for both $D_{5,2}$ and $D_{5,2}^{*}$.

For iterated primitive ideals one can introduce the corresponding torsion number

$$
\lambda_{r}:=\operatorname{dim}_{\mathbb{C}} \frac{\int_{\mathfrak{h}}^{(r)} \mathfrak{g}}{\mathfrak{g}^{r+1}+\mathfrak{h}}, \quad r=0,1, \ldots
$$

Clearly, $\lambda_{1}=\lambda$.

A calculation shows that $\lambda_{2}=4$ for $D_{5,2}$ and 5 for $D_{5,2}^{*}$. Therefore, these higher invariants distinguish between the two singularities. We hope to pursue this line of analysis in a future work.

\section{REFERENCES}

[1] A. Grothendieck and J. Dieudonné, Élements de Géométrie Algébrique, IV Partie, Publ. Math. I.H.E/S. 32 (1967). MR 39:220

[2] G. Jiang, Functions with non-isolated singularities on singular spaces, Thesis, Universiteit Utrecht, 1997.

[3] G. Jiang, D. Siersma, Local embeddings of lines in singular hypersurfaces, Ann. Inst. Fourier, Grenoble 49, 4 (1999), 1129-1147.

[4] J.C. McConnell and J.C. Robson, Noncommutative Noetherian rings, John Wiley \& Sons, 1987. MR 89j: 16023

[5] Y. Nakai, High order derivations I, Osaka J. Math., 7 (1970), 1-27. MR 41:8404

[6] R. Pellikaan, Hypersurface singularities and resolutions of Jacobi modules, Thesis, Rijkuniversiteit Utrecht, 1985.

[7] R. Pellikaan, Finite determinacy of functions with non-isolated singularities, Proc. London Math. Soc., (3)57 (1988), 357-382. MR 89i:32014]

[8] K. Saito, Theory of logarithmic differential forms and logarithmic vector fields, J. Fac. Sci. Univ. Tokyo Sect. 1A Math. 27 (1980), 265-291. MR 83h:32023

[9] P. Seibt, Differential filtrations and symbolic powers of regular primes, Math. Z. 166 (1979) 159-164. MR 80g:13001

[10] W. Vasconcelos, Computational Methods in Commutative Algebra and Algebraic Geometry, Algorithms and Computation in Mathematics, Vol. 2, Springer-Verlag, 1998. MR 99c:13048

Department of Mathematics, Jinzhou Normal University, Jinzhou City, Liaoning 121000, People's Republic of China

Departamento de Matemática, CCen, Universidade Federal de Pernambuco, Av. Prof. Luis Freire, 50740-540 Recife, PE, Brazil

E-mail address: aron@dmat.ufpe.br 